\title{
A Selection of Significant Papers from the Past Year as Recommended by the US Endocrine Disease 2006 Advisory Panel
}

\section{Effect of 6-month calorie restriction on biomarkers of longevity, metabolic adaptation, and oxidative stress in overweight individuals: a randomized controlled trial}

Heilbronn L K, de Jonge L, Frisard M I, et al., Pennington CALERIE Team

JAMA (2006);295(13): pp. 1,539-1,548.

Prolonged calorie restriction increases life span in rodents. Whether prolonged calorie restriction affects biomarkers of longevity or markers of oxidative stress, or reduces metabolic rate beyond that expected from reduced metabolic mass, has not been investigated in humans. The trial's objective was to examine the effects of six months of calorie restriction, with or without exercise, in overweight, non-obese (body mass index, 25 to $<30)$ men and women. The findings suggest that two biomarkers of longevity (fasting insulin level and body temperature) are decreased by prolonged calorie restriction in humans and support the theory that metabolic rate is reduced beyond the level expected from reduced metabolic body mass. Studies of longer duration are required to determine if calorie restriction attenuates the aging process in humans.

From mice to men: insights into the insulin resistance syndromes

Biddinger S B, Kahn C R

Annual Review of Physiology (2006);68: pp. 123-158.

The insulin resistance syndrome refers to a constellation of findings, including glucose intolerance, obesity, dyslipidemia, and hypertension, that promote the development of type 2 diabetes, cardiovascular disease, cancer, and other disorders. Defining the pathophysiological links between insulin resistance, the insulin resistance syndrome, and its sequelae is critical to understanding and treating these disorders. Over the past decade, two approaches have provided important insights into how changes in insulin signaling produce the spectrum of phenotypes associated with insulin resistance. First, studies using tissue-specific knockouts or tissue-specific reconstitution of the insulin receptor in vivo in mice have enabled us to deconstruct the insulin resistance syndromes by dissecting the contributions of different tissues to the insulin-resistant state. Second, in vivo and in vitro studies of the complex network of insulin signaling have provided insight into how insulin resistance can develop in some pathways whereas insulin sensitivity is maintained in others. These data, taken together, give us a framework for understanding the relationship between insulin resistance and the insulin resistance syndromes.

\section{Inhaled insulin improves glycemic control when substituted for or added to oral combination therapy in type 2 diabetes: a randomized, controlled trial}

Rosenstock J, Zinman B, Murphy L J, et al.

Annals of Internal Medicine (2005);:143(8): pp. 549-558.

Patients with type 2 diabetes who do not achieve glycemic control with oral agent therapy eventually require insulin. The objective of the trial was to determine the effect on glycemic control of inhaled insulin alone or added to dual oral therapy (insulin secretagogue and sensitizer) after failure of dual oral therapy. This study evaluated only patients with hemoglobin A1c levels of $8 \%$ to $11 \%$ and did not compare inhaled insulin with other insulins or oral therapy except a dual regimen of secretagogue and sensitizer. The 12-week trial showed that inhaled insulin improved overall glycemic control and hemoglobin A1c level when added to or substituted for dual oral agent therapy with an insulin secretagogue and sensitizer. Consistent with other insulin therapies, hypoglycemia and mild weight gain occurred. Pulmonary function showed no between-group differences.
The impact of assessing specimen adequacy and number of needle passes for fine-needle aspiration biopsy of thyroid nodules

Redman R, Zalaznick H, Mazzaferri E L, et al. Thyroid (2006);:16(1): pp. 55-60.

Fine-needle aspiration biopsy (FNAB) of thyroid nodules is a safe, cost-effective procedure but the rates of inadequate cytology specimens range from approximately $1 \%$ to $15 \%$. This study tests the hypothesis that ultrasonographically $(\mathrm{U} / \mathrm{S})$ guided FNAB and onsite assessment of cytology improves the adequacy rate of FNAB. It conclude that U/S-guided FNAB with onsite evaluation of cytology specimens substantially increases the adequacy of cytology specimens and decreases the number of required needle passes, which ultimately reduces patient discomfort and diagnostic errors, thus raising the question as to whether this should eventually become the standard of care.

\section{Adrenal insufficiency in growth hormone-treated patients: a reassessment \\ Rapaport $R$}

Pediatric Endocrinology Reviews (2006):Apr;3 Suppl 2: pp. 328-332.

There is strong evidence that growth hormone $(\mathrm{GH})$-treated patients are at risk of morbidity and mortality from adrenal insufficiency. Mortality due to sudden adrenal crisis is substantially greater than that from Creutzfeldt-Jakob disease (CJD). The risk of death from adrenal insufficiency in children treated with $\mathrm{GH}$ persists into adulthood, even when adult GH deficiency is not apparent. There is also evidence that very few GH-treated patients are being routinely tested for adrenal insufficiency. The importance of careful evaluation and longterm followup of adrenal function in GH-treated patients, with provision of adequate and aggressive replacement therapy when needed, is emphasized. 


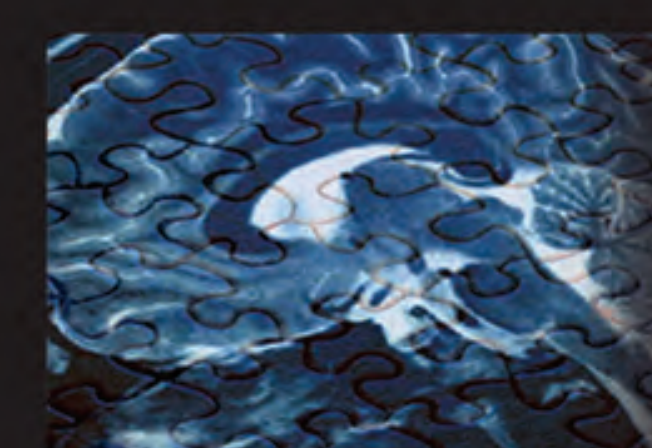
$\hat{S}_{1+1 / n}$
PitutaryNetworkAssociation presents
Co-sponsored byUniversity of California, San Francisco and California Association of Marriage and Family Therapists

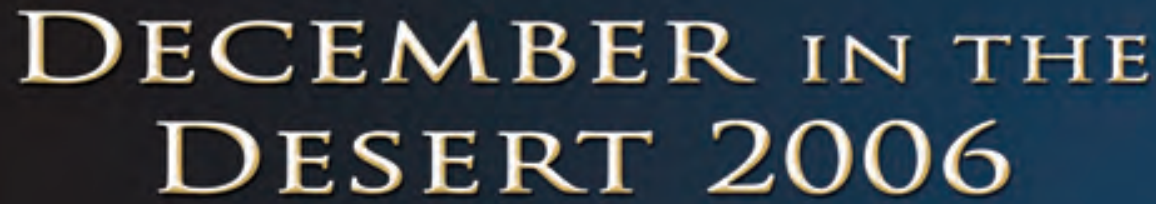

a comprehensive conference covering the many aspects of hormonal health

Dr. Harvey Cushing, the famous pioneering neurosurgeon/physician, had this to say in 1913:

"It is quite probable that the psychopathology of everyday life hinges largely on the discharge of the ductless gland upon the nervous system."

\section{The Pituitary Network Association}

\section{invites you and yours to:}

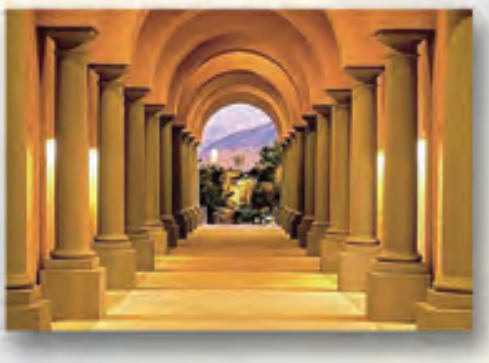

\section{DECEMBER IN THE DESERT 2006}

Conference for Patients and their Families and Physicians,

Nurses, Mental Health Providers, Health Insurance Leaders,

Hospital Administrators and those who "suspect" but still don't know,

as well as the health conscious general public;

December 7 thru 10, at the fabulous Westin Rancho Mirage in Rancho Mirage, California

\section{HEALTH CARE PROVIDERS:}

Do you have patients for whom you can't seem to find answers? They tell of symptoms, but what is the problem? PATIENTS AND FAMILY:

The un-diagnosed, under-diagnosed patient, and the diagnosed patient.

Two Sets of Issues: Medical and Psychological. How do we reconcile the two? Are you at your wits end-tired of looking

The main conference goal is to reduce the time between onset of symptoms and diagnosis so patients can live life to the fullest. Family hormonal meetings will explore patients' symptoms, plus the range of medical, surgical and radiological treatments. And the latest information on long-term follow-up care, maximizing quality of life. We'll delve into the complications that impact physical and emotional health, including mood, fertility, sexual function, diabetes, osteoporosis, and accelerated heart disease.

The conference program will be divided into four segments or Quads, each with two components:

1.) The diagnosed pituitary patient; 2 .) the un-diagnosed or under-diagnosed pituitary patient. Each segment will be led by recognized, highly respected faculty with many years of research and clinical experience in their field. The speakers and presenters will provide unique, often first hand experiences.

Dr. Roger Guillemin, of the Salk Institute in La Jolla, California, winner of the Nobel Prize in medicine, will be one of the featured speakers, and present the topic: "Neuroendocrinology. How did it start and where are we now?" Also, patients' perspectives of pituitary disease will be shared by speakers such as patient advocate Sharmyn McGraw.

New this year will be the addition of mental health professionals as speakers and attendees. Marriage and family therapists, psychologists and other mental health providers are key components to early identification of hormonal difficulties as well as an important part of a good treatment team.

Dr. George Chrousos, Professor of Pediatric Medicine at the University of Athens, Greece and formerly the National Institutes of Health will also be among the featured speakers. He has educated the world regarding pediatric hormonal medicine, Cushing's disease and the mind-body interaction. Dr Chrousos will be honored at the elegant conference banquet Friday evening, as the Gentle Giant for 2006!

Co-hosts California Association of Marriage and Family Therapists, and University Of California, San Francisco will be providing speakers, facilitators and CEU/CME Credits.

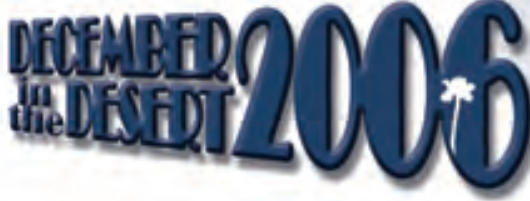

For conference agenda and details, please visit: http://media.pituitary.org/DecDes06/ To register online visit: http://www.pituitarynetwork.org/events/

or contact Barbara Schriber and register today at (805) 499-9973 or barbara@pituitary.org 